\title{
Leber Congenital Amaurosis: Comprehensive Survey of the Genetic Heterogeneity, Refinement of the Clinical Definition, and Genotype-Phenotype Correlations as a Strategy for Molecular Diagnosis
}

\author{
Sylvain Hanein, ${ }^{1}$ Isabelle Perrault, ${ }^{1}$ Sylvie Gerber, ${ }^{1}$ Gaëlle Tanguy, ${ }^{1}$ Fabienne Barbet, ${ }^{1}$ \\ Dominique Ducroq, ${ }^{1}$ Patrick Calvas, ${ }^{2}$ Hélène Dollfus, ${ }^{3}$ Christian Hamel, ${ }^{4}$ Tuija Lopponen, ${ }^{5}$ \\ Francis Munier, ${ }^{6}$ Louisa Santos, ${ }^{7}$ Stavit Shalev, ${ }^{8}$ Dimitrios Zafeiriou, ${ }^{9}$ Jean-Louis Dufier, ${ }^{10}$ \\ Arnold Munnich, ${ }^{1}$ Jean-Michel Rozet, ${ }^{1}$ and Josseline Kaplan ${ }^{1 *}$ \\ ${ }^{1}$ Unité de Recherches sur les Handicaps Génétiques de l'Enfant, Hôpital Necker - Enfants Malades, Paris, France; ${ }^{2}$ Service de Génétique \\ Médicale, Hopital Purpan, Toulouse, France; ${ }^{3}$ Clinique Ophtalmologique, Hopitaux Universitaires de Strasbourg, Strasbourg, France; ${ }^{4}$ Unité \\ INSERM U-254, Montpellier, France; ${ }^{5}$ Clinical Genetics Unit, Turku University Hospital, Turku, Finland; ${ }^{6}$ Hopital Ophtalmique Jules Gonin, \\ Lausanne, Switzerland; ${ }^{7}$ Serviço de Genetica, Hospital Dona Estefânia, Lisboa, Portugal; ${ }^{8}$ Technion - Israel Institute of Technology, Haifa, Israel; \\ ${ }^{9} 1$ st Department of Pediatrics, Aristote University, Thessaloniki, Greece; ${ }^{10}$ Service d'ophtalmologie, Hôpital Necker, France
}

Communicated by Jean-Claude Kaplan

Leber congenital amaurosis (LCA) is the earliest and most severe form of all inherited retinal dystrophies, responsible for congenital blindness. Disease-associated mutations have been hitherto reported in seven genes. These genes are all expressed preferentially in the photoreceptor cells or the retinal pigment epithelium but they are involved in strikingly different physiologic pathways resulting in an unforeseeable physiopathologic variety. This wide genetic and physiologic heterogeneity that could largely increase in the coming years, hinders the molecular diagnosis in LCA patients. The genotyping is, however, required to establish genetically defined subgroups of patients ready for therapy. Here, we report a comprehensive mutational analysis of the all known genes in 179 unrelated LCA patients, including 52 familial and 127 sporadic (27/127 consanguineous) cases. Mutations were identified in $47.5 \%$ patients. GUCY2D appeared to account for most LCA cases of our series (21.2\%), followed by CRB1 (10\%), RPE65 (6.1\%), RPGRIP1 (4.5\%), AIPL1 (3.4\%), TULP1 (1.7\%), and CRX (0.6\%). The clinical history of all patients with mutations was carefully revisited to search for phenotype variations. Sound genotype-phenotype correlations were found that allowed us to divide patients into two main groups. The first one includes patients whose symptoms fit the traditional definition of LCA, i.e., congenital or very early cone-rod dystrophy, while the second group gathers patients affected with severe yet progressive rodcone dystrophy. Besides, objective ophthalmologic data allowed us to subdivide each group into two subtypes. Based on these findings, we have drawn decisional flowcharts directing the molecular analysis of LCA genes in a given case. These flowcharts will hopefully lighten the heavy task of genotyping new patients but only if one has access to the most precise clinical history since birth. Hum Mutat 23:306-317, 2004 @ 2004 Wiley-Liss, Inc.

KEY wORDS: Leber congenital amaurosis; LCA; GUCY2D; retGC1; RPE65; CRX; AIPL1; RPGRIP1; CRB1; TULP1; phenotype-genotype; correlations

DATABASE:

Multigenic disorder: LCA - OMIM: 204000

\section{INTRODUCTION}

Originally described by Theodore Leber in 1869, Leber congenital amaurosis (LCA; MIM\# 204000) is the earliest and most severe form of all hereditary retinal dystrophies, responsible for congenital blindness [Leber, 1869]. The diagnosis is usually made at birth or during the first months of life in an infant with total blindness or greatly impaired vision, normal fundus, and non-recordable electroretinogram (ERG) [Franceschetti and Dieterle,

The Supplementary Material referred to in this article can be found at http://www.mrw.interscience.wiley.com/suppmat/1059-7794/ suppmat/

Received 31 July 2003; accepted revised manuscript 25 November 2003

Grant sponsors: Association Retina France; Association Valentin Haüy; Foundation Fighting Blindness.

Sylvain Hanein and Isabelle Perrault contributed equally to this paper.

*Correspondence to: Josseline Kaplan, Unité de Recherches sur les Handicaps Génétiques de l'Enfant. Hôpital Necker - Enfants Malades, 149 rue de Sèvres, 75743 Paris Cedex 15, France.

E-mail: kaplan@necker.fr

DOI 10.1002/humu.20010

Published online in Wiley InterScience (www.interscience.wiley.com) 
1954]. It was usually accepted that LCA accounted for $5 \%$ of all inherited retinal dystrophies [Kaplan et al., 1990]. Nevertheless, this frequency is underestimated because it is now admitted that in some cases LCA could represent the extreme end of a spectrum of severity of retinal dystrophies [Perrault et al., 2000; Lorenz et al., 2000]. Hitherto, LCA was considered as an autosomal recessive genetically heterogeneous condition. Ten LCA genes have been so far identified or mapped, namely: 1) the retinal specific guanylate cyclase gene (GUCY2D; retGC1; MIM\# 600179) at the LCA1 locus (17p13.1) [Perrault et al., 1996], 2) the gene encoding the $65-\mathrm{kD}$ protein specific to the retinal pigment epithelium (RPE65; MIM\# 180069) at the LCA2 locus (1p31) [Marlhens et al., 1997; Perrault et al., 1999], 3) the cone-rod homeobox-containing gene (CRX, 19q13.3; MIM\# 602225 [Freund et al., 1998; Jacobson et al., 1998; Swaroop et al., 1999], 4) the gene encoding the arylhydrocarbon receptor interacting protein-like 1 (AIPL1; MIM\# 604392) at the LCA4 locus (17p13.1) [Sohocki et al., 2000a], 5) the gene encoding the retinitis pigmentosa GTPase regulator-interacting protein 1 (RPGRIP1; MIM\# 605446) at the LCA6 locus (14q11) [Dryja et al., 2001; Gerber et al., 2001], 6) the human homologue of the Drosophila melanogaster crumbs gene (CRB1, 1q31; MIM\# 604210) [Lotery et al., 2001; den Hollander et al., 2001; Gerber et al., 2002], 7) the gene encoding the tubby-like protein 1 (TULP1, 6q21.3; MIM\# 602280) [North et al., 1997], 8) LCA3 (MIM\# 604232) on chromosome 14q24 [Stockton et al., 1998], 9) LCA5 (MIM\# 604537) on chromosome 6q11-16 [Dharmaraj et al., 2000], and 10) LCA9 on chromosome 1p36 [Keen et al., 2003]. The last three loci, respectively, account for the disease in a consanguineous Saudi Arabian LCA family, a multigenerational kindred of Old Order River Brethren, an isolate hailing from Swiss immigrants to America in the 1750s [Stockton et al., 1998; Dharmaraj et al., 2000], and finally a consanguineous family of Pakistani origin [Keen et al., 2003]. All together, these genes are consistent with autosomal recessive inheritance in the vast majority of cases.

However, some exceptional autosomal dominant forms of LCA associated with heterozygote mutations of the CRX gene have been reported [Tzekov et al., 2001; Perrault et al., 2003]. Interestingly, all LCA genes hitherto identified are involved in strikingly different physiologic pathways resulting in an unforeseeable physiopathologic variety. This outstanding genetic and physiologic diversity that could largely increase in the coming years already hinders the molecular diagnosis in LCA patients. Sound phenotype-genotype correlations are required to lighten the task of molecular biologists, in guiding genetic studies in a new patient, and to anticipate the final outcome in a blind infant.

Here, we report the comprehensive mutation analysis of known LCA genes in a cohort of 179 unrelated LCA patients and the identification of sound phenotypegenotype correlations allowing us to draw decisional flowcharts useful for directing the molecular diagnosis of selected LCA genes.

\section{Patient Panel \\ MATERIALS AND METHODS}

A total of 179 unrelated patients were either seen at the Ophthalmo-genetic Center of Necker Enfants-Malades Hospital or sent to the laboratory by referent ophthalmologists or geneticists from France or other countries worldwide.

Our inclusion criteria were: 1) severe impairment of visual function detected at birth or during the first months of life with pendular nystagmus, roving eye movements, eye poking, inability to follow light or objects, and normal fundus; 2) extinguished ERG; and 3) exclusion of ophthalmological or systemic diseases sharing features with LCA. Detailed clinical data were required for each patient, i.e., 1) age and mode of onset, 2) light behavior since birth, 3) natural history of the visual impairment since the first months of life including the subjective impressions of parents, 4) refraction data, 5) ophthalmologic findings (anterior chamber and fundus), 6) visual acuity (if measurable), and 7) electrophysiology recordings. The course of the disease was determined by interviewing the patients or their parents, and a pedigree was established. Among the 179 families, 52 were multiplex (26 consanguineous) and 127 were simplex cases (27 consanguineous). These data, as well as the origin of the families, are shown in Table 1.

Genomic DNA was extracted from whole blood or immortalized lymphoblast cell lines of patients using standard methods. When a mutation was identified, we examined the parents and other family members when available in both sporadic and familial cases.

\section{Control Panel}

Genomic DNA obtained from 96 unrelated healthy individuals were used as a control panel for molecular studies.

\section{Linkage Analysis of LCA Loci}

For the 10 hitherto identified LCA loci, fluorescent primers flanking markers containing short tracks of di-, tri-, tetra-, or pentanucleotide repeats were either chosen from the Généthon Linkage Map [Dib et al., 1996] or designed from the Working Draft of the Human Genome available at UCSC (Supplementary Table S1, available online at http://www.mrw.interscience.wiley. com/suppmat/1059-7794/suppmat/). In addition, primers flanking intragenic polymorphic markers were designed from the AIPL1, CRX, CRB1, RPGRIP1, and RPE65 sequences, respectively (Supplementary Table S1). Amplified fragments were electrophoresed on an automatic sequencer (ABI 3100, Applied Biosystems, Foster City, CA) and analyzed using the GeneScan Analysis 3.7 and Genotyper softwares.

For each marker, the heterozygote frequency and the size range of alleles were either available from Généthon Linkage Map [Dib et al., 1996] or determined by the study of 50 control individuals (100 chromosomes, see Supplementary Table S1).

\section{Mutational Screening of LCA Genes}

Mutational screening of the seven LCA genes (GUCY2D, RPE65, CRX, AIPL1, RPGRIP1, TULP1, and CRB1) was performed on genomic DNA from the patients using primers designed to flank the splice junctions of each coding exon (Supplementary Table S2A-G, available online at http:// www.mrw.interscience.wiley.com/suppmat/1059-7794/suppmat/). After standard PCR amplification (conditions available on request), products were screened for mutations using denaturing high-pressure liquid chromatography (DHPLC). Heteroduplex formation was induced by heat denaturation of PCR products at $94^{\circ} \mathrm{C}$ for $10 \mathrm{~min}$, followed by gradual reannealing from $94^{\circ} \mathrm{C}$ to $25^{\circ} \mathrm{C}$ over $30 \mathrm{~min}$. DHPLC analysis was performed with the 
TABLE 1. Origin and Genealogy of LCA Families*

\begin{tabular}{|c|c|c|c|c|}
\hline \multirow[b]{2}{*}{ Origin } & \multicolumn{2}{|c|}{ Simplex } & \multicolumn{2}{|c|}{ Multiplex } \\
\hline & Consanguineous & Non-consanguineous & Consanguineous & Non-consanguineous \\
\hline France & 4 & 60 & 2 & 22 \\
\hline North Africa & 11 & 10 & $1 \overline{6}$ & 1 \\
\hline Spain & & 2 & & \\
\hline Benin & 1 & & & \\
\hline Mali & 1 & & & \\
\hline India & & 2 & & \\
\hline Turkey & 4 & & & \\
\hline Italy & 2 & 7 & & 2 \\
\hline Pakistan & 1 & & & \\
\hline Israel & & & 5 & \\
\hline U.K & & 4 & 1 & \\
\hline Greece & 1 & & 1 & \\
\hline Belgium & 1 & 3 & & \\
\hline Senegal & & 1 & & \\
\hline Portugal & & 5 & & \\
\hline Chine & & & 1 & \\
\hline Lebanon & 1 & & & \\
\hline West Indies & 1 & & & \\
\hline Finland & & 4 & & 1 \\
\hline United States & & 1 & & \\
\hline Total & 27 & 100 & 26 & 26 \\
\hline
\end{tabular}

*For each country, the numeric data indicate the number of unrelated families of each genetic type.

WAVE DNA fragment analysis system (Transgenomic, Cheshire, UK). PCR products were eluted at a flow rate of $0.9 \mathrm{ml} / \mathrm{min}$ with a linear acetonitrile gradient. The values of the buffer gradients (buffer A, 0.1 M triethylammoniumacetate; buffer B, $0.1 \mathrm{M}$ triethylammoniumacetate $/ 25 \%$ acetonitrile), start and end points of the gradient, and melting temperature predictions were determined by the WAVEMAKER software (Transgenomic, Cheshire, UK). Optimal run temperatures were empirically determined. Mobile-phase temperatures were assessed within a $5^{\circ} \mathrm{C}$ window above and below the suggested run temperature on the basis of each fragment's characteristic melting profile.

PCR fragments displaying DHPLC abnormal profiles were further sequenced using the Big Dye Terminator Cycle Sequencing Kit v2 (ABI Prism, Applied Biosystems, Foster City, CA on a 3100 automated sequencer).

\section{Mutation Nomenclature}

We have chosen to number the A of the start codon (ATG) of the GenBank cDNA sequences as nucleotide +1 for the genes as follows: GUCY2D, NM_000180; RPE65, NM_000329; CRX, NM_000554; AIPL1, NM_014336; RPGRIP1, NM_020366, CRB1, NM_012076, TULP1, NM_003322.

\section{RESULTS}

\section{Markers for Linkage Study of the 10 LCA Loci}

In addition to available AFM markers [Dib et al., 1996], we have designed new microsatellite markers either intragenic $(\mathrm{n}=4)$, RPE65, AIPL1, CRB1, and RPGRIP1, or extragenic $(n=4)$ flanking the GUCY2D, RPE65, CRX and TULP1 gene (Supplementary Table S1).

The percentage of heterozygosity of designed markers has been calculated by studying 100 chromosomes of diverse geographical origins. All markers but one were highly informative ( $>71 \%$, see Supplementary Table S1).

\section{Spectrum of Mutations of the Seven LCA Genes}

A total of 179 unrelated LCA patients, 52 familial (26 consanguineous), and 127 sporadic cases (27 consangui- neous) were studied. The linkage status of all familial cases and sporadic consanguineous cases was determined using two flanking markers and, when available, an intragenic marker, specific to the 10 LCA loci, respectively. Hints of linkage allowed us to direct the mutational screening of LCA genes in only 13 of 52 familial cases and 6 of 27 consanguineous sporadic cases. For the remaining families and the non-consanguineous sporadic cases, all known genes were screened for mutations.

A total of 85 of 179 (27 familial, 17 consanguineous sporadic, 42 sporadic) were found to carry mutations in one of the seven LCA genes. The respective frequencies of mutations in each gene were, in decreasing order: GUCY2D 21.2\% (38/179 families), CRB1 10\% (18/179 families), RPE65 6.1\% (11/179 families), RPGRIP1 4.5\% (8/179 families), AIPL1 3.4\% (6/179 families), TULP1 $1.7 \%$ (3/179 families), and CRX $0.6 \%$ (1/179 families). All patients but eight were found to be either homozygote for the mutation or compound heterozygote. Mutations are described for each gene in Table 2A-G and graphically in Supplementary Figure S1A-G (available online at http://www.mrw.interscience .wiley.com/suppmat/1059-7794/suppmat/).

GUCY2D (Table 2A, Supplementary Figure S1A). Seventy-five GUCY2D disease-associated mutations were identified in 38 patients with LCA (75/76 disease alleles). A total of 21 patients were found to carry two null GUCY2D alleles (20 homozygous), five patients carried a null allele and a missense mutation, 10 patients harbored two missense mutations (nine homozygous), and one patient was homozygous for an inframe duplication of $48 \mathrm{bp}$.

Thirty-four different GUCY2D mutations were found. Missense mutations make up the majority (16/34), followed by splice and frameshift mutations (7/34, 
respectively), nonsense mutations (3/34), and one inframe duplication. Five of these mutations are novel, while 29 have been reported previously [Perrault et al., 1996; El-Shanti H et al., 1999; Lorenz et al., 2000; Perrault et al., 2000; Rozet et al., 2001; Hanein et al., 2002; Lotery et al., 2003].

Five different alleles account for 36 of the 75 identified disease alleles (47.3\%): 1) the c.387delC mutation was found in a homozygous state in seven unrelated patients, all born to consanguineous parents hailing from North Africa; 2) the c.2943delG deletion was identified in five unrelated patients of Finnish origin (four homozygotes) suggesting a founder effect [Hanein et al., 2002]; 3) the p.Phe565Ser missense mutation was found to homozygously segregate in three unrelated patients of three Algerian families; 4) the c.620delC mutation was identified in two apparently unrelated consanguineous Northern African families; and finally, 5) the p.Ser448X nonsense mutation concerned two unrelated families (one consanguineous) hailing from Italy and Portugal, respectively.

The majority of disease alleles identified in the GUCY2D group of LCA patients are predicted to be null alleles $(47 / 79,61.8 \%)$.

Most mutations were located in exon $2(n=9,17$ families), exon 17 ( $n=4$, four families), exon $15(n=3$, seven families), and intron 9 ( $\mathrm{n}=3$, three families). No mutations were found in exons 5, 6, 10, 11, 13-15, 18, and 19 (Supplementary Figure S1).

RPE65 mutations (Table 2B, Supplementary Figure S1B). Nineteen RPE65 disease alleles were found in 11 unrelated patients (19/22 disease alleles). Four patients harbored a null mutation on each RPE65 allele (two homozygotes, two compound heterozygotes). Three other patients carried two missense mutations (two homozygotes, one compound heterozygote). One patient carried a null mutation on an allele and a missense mutation on the other. Finally, in three patients, only one disease-associated mutation was found (two null and one missense, respectively).

The 16 different RPE65 mutations identified in our series included two nonsense mutations, two one- or two-base pairs deletions, five splice site mutations, and seven missense mutations; none was recurrent. Three of these mutations are new, while 12 have been reported previously [Marlhens et al., 1997; Gu et al., 1997; Morimura et al., 1998; Perrault et al., 1999; Simovich et al., 2001].

The mutations were found to be relatively homogeneously spared throughout the sequence.

CRX mutations (Table 2C, Supplementary Figure S1C). A heterozygous CRX 1bp deletion (c.510delT) was found to segregate through two generations in a family affected with true autosomal dominant LCA [Perrault et al., 2003]. This mutation located in the third exon of the gene led to the apparition of a premature stop codon and the truncation of three important protein domains, including the OTX tail [Perrault et al., 2003].

AIPL1 mutations (Table 2D, Supplementary Figure S1D). In 6 of 179 unrelated patients with LCA, 12 disease-associated alleles were identified. Four patients were found to carry a null mutation deleting the hinge domain on each AIPL1 allele. Indeed, the p.Trp278X mutation was found to be homozygous in three simplex patients born to non-consanguineous parents hailing from France $(n=2 / 3)$ and Spain $(1 / 3)$, respectively. In a fourth non-consanguineous family, this same mutation was found to be associated in patients with a splice-site mutation (c.277-2A>G).

In addition, two patients were found to be homozygous for a missense mutation converting an amino acid of one class into an amino acid of another class. The p.Val71Phe mutation was identified in a non-consanguineous simplex case while the pAla197Pro mutation was found to segregate in a consanguineous LCA family originating from North Africa.

Three of the four different AIPL1 mutations described here, including the recurrent p.Trp278X mutation (7/12 disease alleles, 58\%), have already been reported elsewhere [Sohocki et al., 2000].

RPGRIP1 mutations (Table 2E, Supplementary Figure S1E). Twelve RPGRIP1 disease alleles were found in 8 of 179 unrelated LCA patients Among these patients, 2 of 8 carried homozygous mutations ( 2 of 2 familial consanguineous), 2 of 8 were compound heterozygotes ( 2 of 2 simplex non-consanguineous), and four were single heterozygotes (4 of 4 simplex non-consanguineous).

A total of 10 different mutations were found, all but one being null alleles: four small frameshift insertions or deletions, two splice mutations, and three nonsense mutations. All nine mutations are expected to delete the RPGR interacting domain. Only one missense mutation converting a conserved uncharged amino acid into an acidic amino acid was found in a patient born to consanguineous parents hailing from Morocco (p.Gly746Glu). This amino acid is conserved in murine and bovine sequences and lies in the coiled-coiled domain of the protein. Of the 10 different RPGRIP1 mutations, three are novel and seven have already been reported [Dryja et al., 2001; Gerber et al., 2001].

CRB1 mutations (Table 2F, Supplementary Figure S1F). In 18 of 179 unrelated LCA patients, 36 diseaseassociated mutations were found. Nine of these 18 patients were found to be homozygote for null alleles ( $\mathrm{n}$ $=4$, three sporadic) or missense mutations $(\mathrm{n}=5$, three sporadic). The nine others patients were either compound heterozygotes for a null mutation and a missense mutation ( $\mathrm{n}=5$, five sporadic), or two missense mutations ( $n=4$, eight sporadic).

A total of 21 different CRB1 mutations were found including three nonsense mutations, five frameshift microdeletions, one splice mutation, and 12 missense mutations. Ten of these mutations are novel while 11 have already been reported [Lotery et al., 2001; den Hollander et al., 2001; Gerber et al., 2002]. All mutations but one were scattered over exons 6 to 12 .

Three different mutations were found in more than one family. The p.Cys948Tyr missense mutation was identified in three French simplex cases. One of them, born to consanguineous parents, harbored the mutation 
TABLE 2

\begin{tabular}{|c|c|c|c|c|c|c|}
\hline Family & Exon & Base change & Allele 1 predicted change & Exon & Base change & Allele 2 predicted change \\
\hline \multicolumn{7}{|c|}{ A. GUCY2D Mutations in Patients Affected With LCA ${ }^{\dagger}$} \\
\hline $3 \mathrm{~F}^{*}$ & 2 & c. $387 \mathrm{delC}$ & p.Asn $129 \mathrm{fs} \times 165^{1,2,3}$ & 2 & c.387 delC & p.Asn129fsX165 $1,2,3$ \\
\hline $7 \mathrm{~F}^{*}$ & 8 & c. $1694 \mathrm{~T}>\mathrm{C}$ & p.Phe565Ser ${ }^{2}$ & 8 & c. $1694 \mathrm{~T}>\mathrm{C}$ & p.Phe565Ser ${ }^{2}$ \\
\hline $11 S^{*}$ & 2 & c. $3 G>C$ & p.Met1 $?^{2}$ & 2 & c. $3 G>C$ & p.Met1 $?^{2}$ \\
\hline $17 \mathrm{~F}^{*}$ & 8 & c. $1694 \mathrm{~T}>\mathrm{C}$ & p.Phe565Ser ${ }^{2}$ & 8 & c.1694T $>C$ & p.Phe565Ser ${ }^{2}$ \\
\hline $20 S^{*}$ & 9 & c.1805.1829del & p.Arg602fsX636 ${ }^{2}$ & 9 & c.1805.1829del & p.Arg602fsX636 \\
\hline $23 \mathrm{~F}^{*}$ & 2 & c.620 delC & p.Ala $207 \mathrm{fs} X 215^{1,2}$ & 2 & c.620delC & p.Ala $207 \mathrm{fs} X 215^{1,2}$ \\
\hline $31 S^{*}$ & 15 & c. $2927 \mathrm{G}>\mathrm{T}$ & p.Arg976Leu ${ }^{2}$ & 15 & c. $2927 \mathrm{G}>\mathrm{T}$ & p.Arg 976Leu ${ }^{2}$ \\
\hline $33 S^{*}$ & 17 & c.3078.3079delGA & p.Gly1026fsX1071 2 & 17 & c.3078.3079delGA & p.Gly1026fsX1071 2 \\
\hline $34 S^{*}$ & 2 & c.620delC & p.Ala $207 \mathrm{fs} \times 215^{1,2}$ & 2 & c.620delC & p.Ala207fsX215 \\
\hline $36 S^{*}$ & 8 & c. $1694 \mathrm{~T}>\mathrm{C}$ & p.Phe 565Ser ${ }^{2}$ & 8 & c. $1694 \mathrm{~T}>\mathrm{C}$ & p.Phe565Ser ${ }^{2}$ \\
\hline $51 \mathrm{~F}$ & 2 & c. $3 G>A$ & p.Met1 $?^{2}$ & Intron 9 & c. $1957-1 G>T^{2}$ & Aberrant splicing \\
\hline $52 S$ & 3 & c. $937 \mathrm{C}>\mathrm{T}$ & p.Arg 313 Cys $^{2}$ & 4 & c. $1343 \mathrm{C}>\mathrm{A}$ & p.Ser $448 \mathrm{X}^{2}$ \\
\hline $56 S^{*}$ & 17 & c. $3956 \mathrm{~A}>\mathrm{C}$ & p.His1019Pro & 17 & c. $3956 \mathrm{~A}>\mathrm{C}$ & p.His1019Pro ${ }^{2}$ \\
\hline $70 S^{*}$ & Intron 9 & c. $1956+2 \mathrm{~T}>\mathrm{A}^{2}$ & Aberrant splicing & Intron 9 & c. $1956+2 T>A$ & Aberrant splicing \\
\hline $72 \mathrm{~S}$ & 17 & c. $3106 \mathrm{C}>\mathrm{T}$ & p.Gln $1036 X^{2}$ & 17 & c. $3106 \mathrm{C}>\mathrm{T}$ & p.G $\ln 1036 \mathrm{X}^{2}$ \\
\hline $76 \mathrm{~F}$ & 4 & c. $1052 A>G$ & p.Tyr $351 C y s^{4}$ & 4 & c. $1052 A>G$ & p.Tyr $351 C y s^{4}$ \\
\hline $82 \mathrm{~S}$ & 2 & c. $387 \mathrm{C}>\mathrm{A}$ & p.Asn129 Lys ${ }^{2}$ & 16 & c. $2983 \mathrm{C}>\mathrm{T}$ & p.Arg $995 \operatorname{Trp}^{2}$ \\
\hline $85 S^{*}$ & 2 & c.52.99dup & p.Gly18.Leu33dup ${ }^{2}$ & 2 & c.52.99dup & p.Gly18.Leu 33dup ${ }^{2}$ \\
\hline $88 \mathrm{~S}$ & 16 & c. $3025 \mathrm{~A}>\mathrm{T}$ & p.Met1009Leu ${ }^{2}$ & Intron 9 & c. $1957-1 G>T^{2}$ & Aberrant splicing \\
\hline $89 \mathrm{~F}$ & 2 & c. $121 \mathrm{C}>\mathrm{T}$ & p.Leu41Phe & 2 & c.121C $>\mathrm{T}$ & p.Leu41Phe \\
\hline $90 \mathrm{~F}^{*}$ & 2 & c.387 delC & p.Asn $129 \mathrm{fs} X 165^{1,2,3}$ & 2 & c. $387 \mathrm{delC}$ & p.Asn129fsX165 $1,2,3$ \\
\hline $91 \mathrm{~F}^{*}$ & 2 & c.387 delC & p.Asn129fsX165 $1,2,3$ & 2 & c. $387 \mathrm{delC}$ & p.Asn129fsX165 $1,2,3$ \\
\hline $110 S^{*}$ & 2 & c. $226.239 \mathrm{del}$ & PAla 76fsX84 & 2 & c. $226.239 \mathrm{del}$ & pAla76fs $\times 84^{2}$ \\
\hline $113 S$ & 4 & c. $1343 \mathrm{C}>\mathrm{A}$ & p.Ser $448 X^{2}$ & 4 & c. $1343 \mathrm{C}>\mathrm{A}$ & p.Ser $448 \mathrm{X}^{2}$ \\
\hline $117 \mathrm{~S}$ & 7 & c. $1618 \mathrm{C}>\mathrm{T}$ & p.Arg540Cys ${ }^{2}$ & & “?” & \\
\hline $121 \mathrm{~S}^{*}$ & 2 & c.387 delC & p.Asn $129 \mathrm{fs} X 165^{1,2,3}$ & 2 & c. $387 \mathrm{delC}$ & p.Asn129fsX165 $1,2,3$ \\
\hline $129 \mathrm{~S}$ & 2 & c.387 delC & p.Asn $129 \mathrm{fs} X 165^{1,2,3}$ & 2 & c.387 delC & p.Asn129fsX165 $1,2,3$ \\
\hline $134 \mathrm{~S}$ & Intron 7 & c. $1566+2 \mathrm{~T}>\mathrm{C}$ & Aberrant splicing & 2 & c. $2 \mathrm{~T}>\mathrm{A}$ & p.Met1 ? ${ }^{2}$ \\
\hline $138 \mathrm{~S}$ & Intron 16 & c. $3043+4 \mathrm{~A}>\mathrm{T}$ & Aberrant splicing & 15 & c.2943delG & p.Ser $981 \mathrm{fs} \times 1071^{5}$ \\
\hline $139 \mathrm{~S}^{*}$ & 17 & c. $3118 \mathrm{C}>\mathrm{T}$ & p.Arg1040X & 17 & c. $3118 \mathrm{C}>\mathrm{T}$ & p. Arg1040X \\
\hline $147 \mathrm{~F}$ & 15 & c.2943delG & p.Ser981fsX1071 & 15 & c. 2943 delG & p.Ser981fsX1071 5 \\
\hline $148 \mathrm{~S}$ & 15 & c.2943delG & p.Ser981fsX1071 ${ }^{5}$ & 15 & c.2943delG & p.Ser $981 \mathrm{fs} X 1071^{5}$ \\
\hline $155 \mathrm{~S}$ & 12 & c. $20302 \mathrm{C}>\mathrm{T}$ & PArg 768Trp & 2 & c.87.88insC & p.Pro $30 \mathrm{fs} X 318^{2}$ \\
\hline $165 S^{*}$ & 2 & c. $387 \mathrm{delC}$ & p.Asn $129 \mathrm{fs} X 165^{1,2,3}$ & 2 & c. $387 \mathrm{delC}$ & p.Asn129fsX165 2,3 \\
\hline $170 S^{*}$ & 2 & c. $387 \mathrm{delC}$ & p.Asn $129 \mathrm{fs} \times 165^{1,2,3}$ & 2 & c. $387 \mathrm{delC}$ & p.Asn129fsX165 2,3 \\
\hline $171 S^{*}$ & 15 & c. $2800 \mathrm{G}>\mathrm{C}$ & p.Ala934Pro & 15 & c. $2800 \mathrm{G}>\mathrm{C}$ & p.Ala934Pro \\
\hline $196 \mathrm{~S}$ & 15 & c.2943delG & p.Ser981fsX1071 5 & 15 & c.2943delG & p.Ser981fsX1071 ${ }^{5}$ \\
\hline $257 \mathrm{~S}$ & 15 & c.2943delG & p.Ser $981 \mathrm{fsX1071^{5 }}$ & 15 & c.2943delG & p.Ser981fsX1071 \\
\hline \multicolumn{7}{|c|}{ B. RPE65 Mutations in Patients Affected With LCA ${ }^{\dagger}$} \\
\hline $1 \mathrm{~F}^{*}$ & Intron 1 & $c .11+5 G>A$ & Aberrant splicing & Intron 1 & $c .11+5 G>A^{6}$ & Aberrant splicing \\
\hline $8 \mathrm{~F}$ & Intron 8 & c. $912+1 G>A^{6}$ & Aberrant splicing & & & "?" \\
\hline $16 \mathrm{~F}$ & 7 & c.700C $>\mathrm{T}$ & p.Arg $234 X^{6}$ & 10 & c.1060delA & p.Lys $354 \mathrm{fs} X 372^{6}$ \\
\hline $29 \mathrm{~S}$ & 10 & c. $1087 \mathrm{C}>\mathrm{A}$ & p.Pro363The $e^{6,7}$ & 13 & c. $1418 \mathrm{~T}>\mathrm{A}$ & p.Val473Asp 8 \\
\hline $54 S^{*}$ & 3 & c. $190 \mathrm{C}>\mathrm{T}$ & p. $G \ln 64 X^{6}$ & 3 & c. $190 \mathrm{C}>\mathrm{T}$ & p.Gln $64 X^{6}$ \\
\hline $95 \mathrm{~S}$ & 12 & c. $1031 \mathrm{~T}>\mathrm{C}$ & p.Ala $434 \mathrm{Val}^{6}$ & & "?" & "?" \\
\hline $114 S^{*}$ & 9 & c. $989 \mathrm{G}>\mathrm{A}$ & p.Cys330Tyr 6 & 9 & c. $989 G>A$ & p.Cys330Tyr 6 \\
\hline $122 \mathrm{~F}^{*}$ & Intron 2 & c. $94-2 \mathrm{~A}>\mathrm{T}$ & Aberrant splicing & Intron 2 & c. $94-2 A>T$ & Aberrant splicing \\
\hline $128 \mathrm{~S}$ & 6 & c. $544 \mathrm{C}>\mathrm{A}$ & p.His182Asn ${ }^{8}$ & Intron 6 & c. $644-2 \mathrm{~A}>\mathrm{T}^{8}$ & Aberrant splicing \\
\hline $182 \mathrm{~F}$ & 3 & c.131G $>A$ & p. Arg 44Gln 9 & 5 & c. $444 \mathrm{G}>\mathrm{T}$ & p.Glu148Asp \\
\hline $213 \mathrm{~F}$ & 6 & c.615.616delCA & p.Asn 205fsX233 & & "?" & \\
\hline \multicolumn{7}{|c|}{ C. CRX Mutations in Patients Affected With $\mathrm{LCA}^{\dagger}$} \\
\hline $22 \mathrm{~F}$ & 3 & c.510deIT & p.Pro170fsX526 ${ }^{10}$ & & & \\
\hline \multicolumn{7}{|c|}{ D. AIPL1 Mutations in Patients Affected with LCA ${ }^{\dagger}$} \\
\hline $6 \mathrm{~S}$ & 6 & c.834G $>\mathrm{A}$ & p.Trp $278 X^{11}$ & 6 & c.834G >A & p.Trp $278 X^{11}$ \\
\hline $24 \mathrm{~F}$ & 6 & c.834G $>\mathrm{A}$ & p.Trp $278 \mathrm{X}^{11}$ & Intron 2 & c. $277-2 \mathrm{~A}>\mathrm{G}$ & Aberrant splicing \\
\hline $26 \mathrm{~F}^{*}$ & 4 & c.589G $>\mathrm{C}$ & p.Ala197Pro & 4 & c. $589 \mathrm{G}>\mathrm{C}$ & p.Ala197Pro \\
\hline $115 \mathrm{~S}$ & 6 & c. $834 \mathrm{G}>\mathrm{A}$ & p.Trp $278 X^{11}$ & 6 & c.834G $>A$ & p.Trp $278 X^{11}$ \\
\hline $149 \mathrm{~S}$ & 6 & c.834G $>A$ & p.Trp $278 X^{11}$ & 6 & c.834G $>A$ & p.Trp $278 X^{11}$ \\
\hline $166 S$ & 2 & c. $211 \mathrm{G}>\mathrm{T}$ & p.Val 71Phe & 2 & c. $211 \mathrm{G}>\mathrm{T}$ & p.Val 71Phe \\
\hline
\end{tabular}

Mutations of a) GUCY2D, b) RPE65, c)CRX, d) AIPL1, e) RPGRIP1, f) CRB1, and g) TULP1 identified in our panel of LCA families. Asterisks indicate consanguinity of the parents of LCA patients. S, sporadic case; F, familial case. The A of the start codon (ATG) of the GenBank cDNA sequences is nucleotide +1 for the genes as follows: GUCY2D, NM.000180; RPE65, NM.000329; CRX, NM.000554; AIPL1, NM.014336; RPGRIP1, NM.020366, CRB1, NM.012076; TULP1, NM.003322.

References: ${ }^{1}$ Perrault et al. [1996]; ; ${ }^{2}$ Perrault et al. [2000]; Lorenz et al. [2000]; ${ }^{3}$ Rozet et al. [2001]; ${ }^{4}$ Lotery et al. [2003]; ${ }^{5}$ Hanein et al. [2002]; ${ }^{6}$ Marlhens et al. [1997]; Perrault et al. [1999]; ${ }^{7}$ Gu et al. [1997]; ${ }^{8}$ Lorenz et al. [2000]; ${ }^{9}$ Simovich et al. [2001]; ${ }^{10}$ Perrault et al. [2003]; ${ }^{11}$ Sohocki et al. [2000]; ${ }^{12}$ Dryja et al. [2001]; Gerber et al. [2001]; ${ }^{13}$ Lotery et al. [2001]; ${ }^{14}$ den Hollander et al. [2001]; ${ }^{15}$ Gerber et al. [2002]. 
TABLE 2.(Continued)

\begin{tabular}{|c|c|c|c|c|c|c|}
\hline Family & Exon & Base change & Allele 1 predicted change & Exon & Base change & Allele 2 predicted change \\
\hline \multicolumn{7}{|c|}{ E. RPGRIP1 Mutations in Patients Affected With LCA ${ }^{\dagger}$} \\
\hline $4 \mathrm{~F}^{*}$ & 15 & c. $2237 \mathrm{G}>\mathrm{A}$ & p.Gly746Glu ${ }^{12}$ & 15 & c. $2237 \mathrm{G}>\mathrm{A}$ & p.Gly $746 \mathrm{Glu}^{12}$ \\
\hline $9 S$ & Intron 8 & c. $490+3 A>G^{12}$ & Aberrant splicing & & "?" & "?" \\
\hline $46 \mathrm{~S}$ & 12 & c.1501.1502insTGTC & p.Leu501fsX508 ${ }^{12}$ & & “?” & “?” \\
\hline $77 \mathrm{~s}$ & 16 & c. $2534 \mathrm{C}>\mathrm{T}$ & p. Arg 852X & intron 16 & c. $2710+1 G>A$ & Aberrant splicing \\
\hline $104 \mathrm{~F}^{*}$ & 4 & c.510deIT & p.Tyr170fsX189 12 & 4 & c.510delT & p.Tyr170fsX189 12 \\
\hline $126 \mathrm{~S}$ & 21 & c. $3341 \mathrm{~A}>\mathrm{G}$ & p.Asp1114Gly & 16 & c. $2668 \mathrm{C}>\mathrm{T}$ & p.Arg $890 \mathrm{X}$ \\
\hline $160 \mathrm{~S}$ & 12 & c. $1525 \mathrm{C}>\mathrm{T}$ & p. $G \ln 509 X^{12}$ & & "?" & "?" \\
\hline $167 \mathrm{~S}$ & 16 & c.2576.2577 insTT & p.Ser858fsX865 12 & 23 & c.3629.3630insG & p.The1210fsX1214 ${ }^{12}$ \\
\hline \multicolumn{7}{|c|}{ F. CRB1 mutations in Patients Affected With LCA ${ }^{\dagger}$} \\
\hline $2 \mathrm{~F}^{*}$ & 6 & c. $2128 \mathrm{G}>\mathrm{C}$ & p.Glu 710Gln ${ }^{13}$ & 6 & c. $2128 \mathrm{G}>\mathrm{C}$ & p.Glu 710Gln 13 \\
\hline $2.1 \mathrm{~F}$ & 9 & c. $3320 \mathrm{~T}>\mathrm{G}$ & p.Leu1107 Arg ${ }^{13}$ & 9 & c. $3320 \mathrm{~T}>\mathrm{G}$ & p.Leu1107 Arg ${ }^{13}$ \\
\hline $2.2 \mathrm{~F}$ & 9 & c. $3320 \mathrm{~T}>\mathrm{G}$ & p.Leu1107Arg 13 & 6 & c. $2128 \mathrm{G}>\mathrm{C}$ & p.Glu $710 \mathrm{Gln}^{13}$ \\
\hline $14 \mathrm{~S}$ & 9 & c. $3320 \mathrm{~T}>\mathrm{G}$ & p.Leu1107 Arg ${ }^{13}$ & 9 & c. $3320 \mathrm{~T}>\mathrm{G}$ & p.Leu1107 Arg ${ }^{13}$ \\
\hline $41 S^{*}$ & 9 & c. $2843 \mathrm{G}>\mathrm{A}$ & p.Cus 948 Tur $^{13,14}$ & 9 & c. $2843 \mathrm{G}>\mathrm{A}$ & p.Cus 948 Tyr $^{13,14}$ \\
\hline $42 S$ & 8 & c. $2688 \mathrm{~T}>\mathrm{A}$ & p.Cys896X & 8 & c. $2688 \mathrm{~T}>\mathrm{A}$ & p.Cys $896 \mathrm{X}^{14}$ \\
\hline $54.1 \mathrm{~S}$ & 9 & c. $2843 G>T$ & p.Cys948Tyr & 11 & c. $3961 \mathrm{~T}>\mathrm{A}$ & p.Cys1321Gly \\
\hline $80 \mathrm{~F}^{*}$ & 12 & c.4121.4130del & p.Arg1374fsX1397 & 12 & c.4121.4130del & p.Arg1374fsX1397 15 \\
\hline $124 \mathrm{~S}$ & 9 & c.2853.2854insT & p.Ala $952 \mathrm{fs} \times 972$ & 9 & c.2853.2854insT & p.Ala952fsX972 \\
\hline $131 \mathrm{~S}$ & 7 & c. $2222 \mathrm{~T}>\mathrm{C}$ & p.Met741Thr 13 & 11 & $\begin{array}{l}\text { c.3988delG } \\
\text {. }\end{array}$ & p.Glu1330fsX1340 \\
\hline $154 \mathrm{~S}$ & 9 & c. $3307 \mathrm{G}>\mathrm{A}$ & p.Gly1103Arg & 9 & c.3345delT & p.Gly1115fsX1140 \\
\hline $161 S$ & 7 & c. $2234 \mathrm{C}>\mathrm{T}$ & p.Thr $745 \mathrm{Met}^{15}$ & 9 & c. $3074 \mathrm{G}>\mathrm{T}$ & p.Ser1025Ile \\
\hline $163 \mathrm{~S}$ & 7 & c. $2555 \mathrm{~T}>\mathrm{C}$ & p.Ile852Thr ${ }^{15}$ & Intron 11 & c. $4005+1 \mathrm{G}>\mathrm{A}$ & Aberrant splicing \\
\hline $164 \mathrm{~S}$ & 9 & c. $3074 \mathrm{G}>\mathrm{T}$ & p.Ser1025Ile & 9 & c. $3320 \mathrm{~T}>\mathrm{C}$ & p.Leul107 Pro ${ }^{15}$ \\
\hline $187 \mathrm{~S}$ & 2 & c.613.619deITAGGAAG & p.Ile205fsX219 & 9 & c. $2843 \mathrm{G}>\mathrm{A}$ & p.Cys 948 Tyr $^{15,16}$ \\
\hline $200 S^{*}$ & 6 & c. $1750 \mathrm{G}>\mathrm{T}$ & $\begin{array}{l}\text { p.Asp 584Tyr } \\
\text { p. }\end{array}$ & 6 & c. $1750 \mathrm{G}>\mathrm{T}$ & p.Asp584Tyr \\
\hline $202 S^{*}$ & 11 & c. $3879 \mathrm{G}>\mathrm{A}$ & p.Trp1293X & 11 & c. $3879 \mathrm{G}>\mathrm{A}$ & p.Trp1293X \\
\hline $206 \mathrm{~S}$ & 7 & c. $2290 \mathrm{C}>\mathrm{T}$ & p.Arg 764Cys ${ }^{16}$ & 7 & c. $2479 \mathrm{G}>\mathrm{T}$ & p.Gly $827 X$ \\
\hline \multicolumn{7}{|c|}{ G.TULP1 Mutations in Patients Affected With LCA ${ }^{\dagger}$} \\
\hline $57 S^{*}$ & 12 & c. $1198 \mathrm{C}>\mathrm{T}$ & p.Arg 400Trp & 12 & c. $1198 \mathrm{C}>\mathrm{T}$ & p.Arg 400Trp \\
\hline $75 \mathrm{~F}$ & Intron 2 & c. $99+1 \mathrm{G}>\mathrm{A}$ & Aberrant splicing & 12 & c. $1204 \mathrm{G}>\mathrm{T}$ & p.Glu402X \\
\hline $240 \mathrm{~F}^{*}$ & 11 & c. $1102 \mathrm{G}>\mathrm{T}$ & p.Gly368Trp & 11 & c. $1102 \mathrm{G}>\mathrm{T}$ & p.Gly368Trp \\
\hline
\end{tabular}

homozygously. In the other two, the p.Cys948Tyr mutation was found to be associated to an other missense mutation (p.Cys1321Gly) and a null allele (c.613_619del), respectively. The p.Leu1107Pro mutation was found to be homozygous in one patient and associated to the p.Ser1025Ile missense mutation in another one, both being French non-consanguineous simplex cases. This latter mutation was also found to be associated with the p.The745Met mutation in an other French simplex patient.

TULP1 mutations (Table 2G, Supplementary Figure S1G). Six disease alleles were identified in 3 of 179 unrelated patients. One patient, belonging to multiplex family originating from Italy, was found to carry a splice mutation (c.99+1G>A) and a nonsense mutation (p.Glu402X). The other two, born to consanguineous parents (one simplex), were homozygous for a missense mutation (p.Arg400Trp, pGly368Trp, respectively). Both missense mutations concern conserved amino acids lying in the very conserved C-terminal region of the TUB family, including TUB and TULP2 [North et al., 1997].

The four different TULP1 mutations identified in our series have not been reported earlier.

Phenotype-genotype correlations. All patients included in this study fulfilled strict inclusion criteria for diagnosis of LCA, i.e., congenital nystagmus, no or very poor ocular pursuit, digito-ocular signs of Franceschetti attesting of profoundly impaired vision, and no recordable ERG since birth or the first months of life. For the vast majority of patients, the screening of all seven LCA genes has been randomly undertaken, especially as the severity of the disease in the early beginning of life appeared to be sufficient to assert the diagnosis of LCA. Among the 179 LCA families belonging to our series, at least one disease-causing mutation was found in one of the seven LCA genes in 85 patients. For each of these patients, the clinical history was strictly revisited by questioning themselves when adults or their parents when younger. When necessary and possible, an ophthalmoscopic examination as well as refraction measurements were performed. The visual field was recorded when the visual acuity allowed it. Brought up to date, these clinical data resulted in the division of LCA patients into two groups on the basis of their light behavior during the two first years of life. Indeed, the patients mainly displayed either photophobia or night blindness. In the group of patients complaining of photophobia, hypermetropia was constantly noted as well as severe involvement of both types of photoreceptor cells leading to early peripheral and macular degeneration of the retina with bone spicule pigments in the periphery, retinal atrophy including the macular region, very thin vessels, and optic disc pallor. When the hypermetropia was very severe, higher than +7 , the 
visual acuity was reduced to counting fingers $(\mathrm{CF})$ or light perception (LP). In these cases, the disease is not progressive and is pathognomonic of GUCY2D mutations [Perrault et al., 1996]. When the hypermetropia was lower than +7 , the visual acuity was frequently countable and ranged from CF and 1/20. These data, as well as the presence of a keratoconus, suggest mutations in the AIPL1 or RPGRIP1 genes. In this first group of patients, the disease comes in the form of congenital or very early cone-rod dystrophy with dramatic and invariable cone dysfunction (Fig. 1).

In the second group of patients complaining from night blindness, two clinical subtypes can be distinguished, one with hypermetropia and the second without hypermetropia.

In the first subtype, an early macular reorganization is almost constantly visible at the fundus examination. Consequently, a central scotoma is noted at the visual field and the visual acuity ranges from $1 / 10$ to $2 / 10$ in the first decade of life. These clinical findings suggest mutations in either CRB1 or CRX. In the second subtype, an early peripheral involvement of the retina is visible at the fundus examination giving an early aspect of retinitis pigmentosa. The visual field confirms this aspect and shows a progressive concentric reduction. The visual acuity is much better than in other groups of patients, especially during daytime, reaching values ranging from $1 / 10$ to $2 / 10$ or better during the first decade. This "moderate" form of LCA strongly suggests mutations in RPE65 or TULP1 genes.

In this second group of patients, the disease comes in the form of a severe yet progressive rod-cone dystrophy, still different from the stationary cone-rod dystrophy of the first group of patients (Fig. 1).

\section{DISCUSSION}

The genetic heterogeneity of Leber congenital amaurosis has been accepted for a long time [Wardenburg et al., 1963] but it turned out to be largely higher than all odds. So far, 10 genes have been mapped on human chromosomes and seven identified. One of the aims of the present study was to determine the prevalence of each genetic subtype by genotyping 179 unrelated LCA patients hailing from countries worldwide. Mutations were identified in 85 of 179 patients (47.5\%). GUCY2D mutations appeared to account for most of the LCA in our series (21.2\%), followed by CRB1 (10\%), RPE65 (6.1\%), RPGRIP1 (4.5\%), AIPL1 (3.4\%), TULP1 (1.7\%), and CRX (0.6\%).

This wide genetic heterogeneity raises a problem of genetic counseling in couples of LCA patients who wish to have children. Indeed, in these situations, the risk for their descent is either null (different genes in patients) or

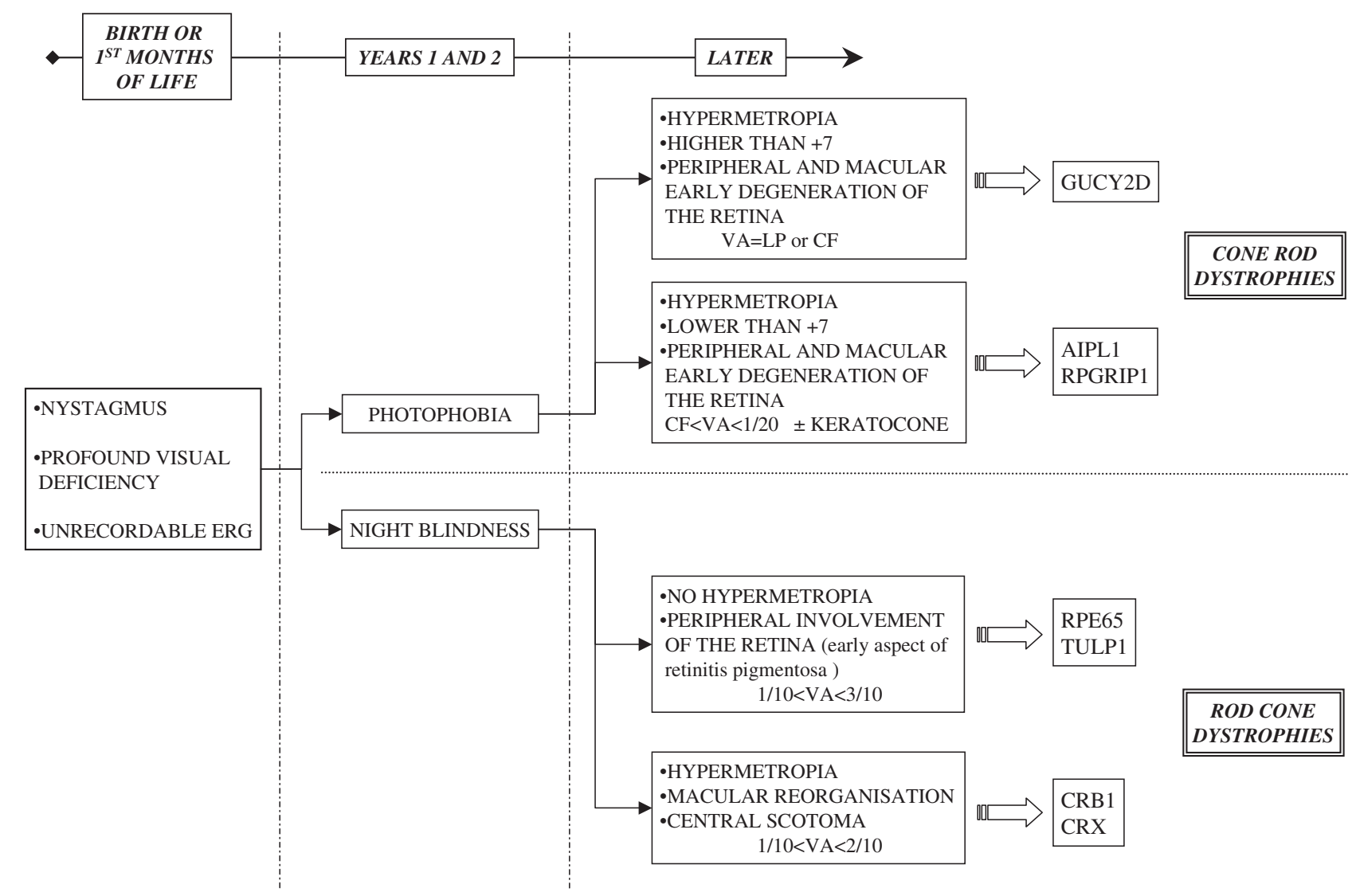

FIGURE 1. Clinical flowchart. LCA and early onset retinitis pigmentosa are divided on the basis of light behavior, the refraction data, the aspect of the retina, and the visual acuity (VA). CF, counting fingers; LP, light perception. This flowchart allows one to direct the molecular analysis of selected LCA genes. 


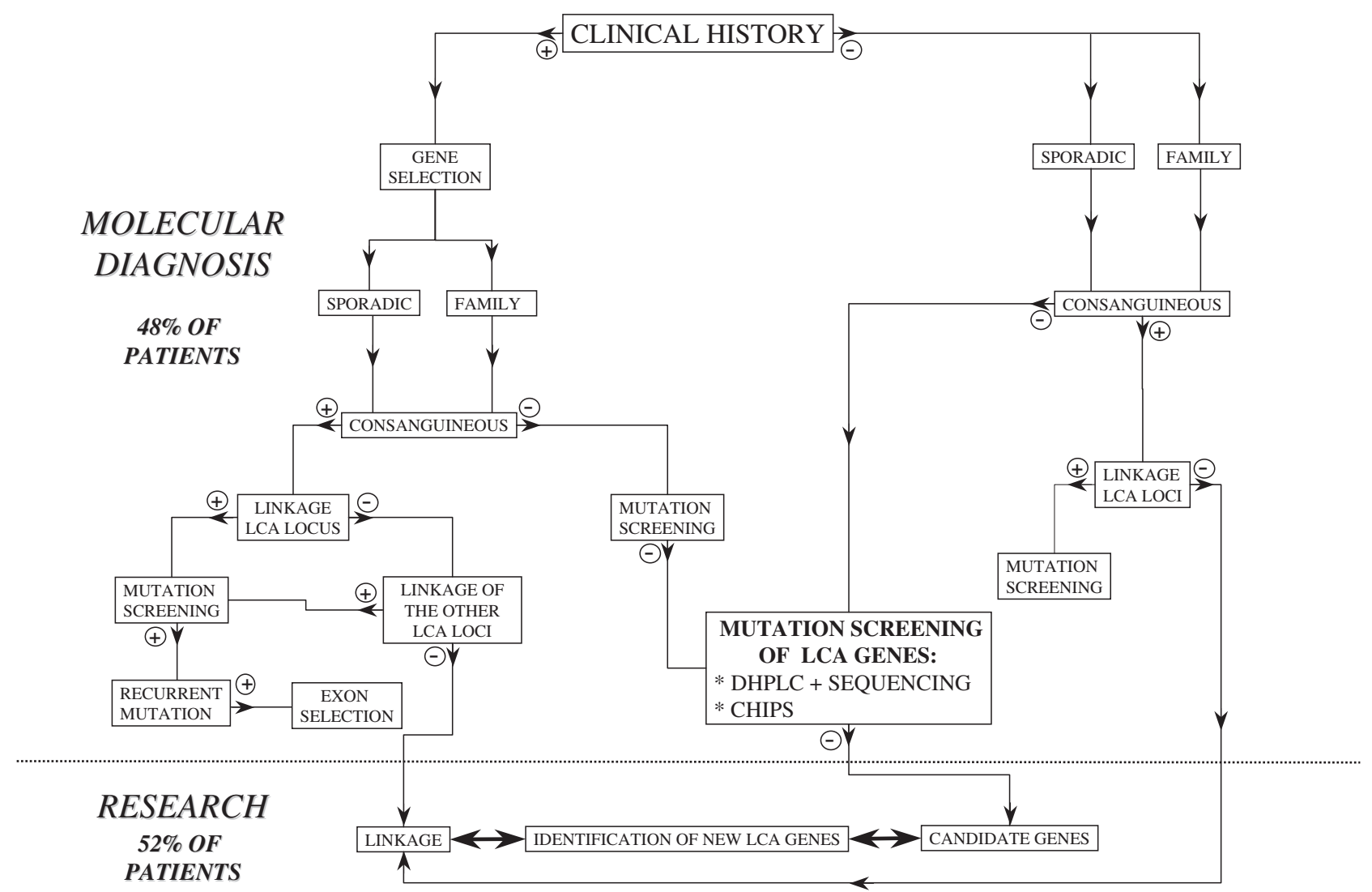

FIGURE 2. Decision-making flowchart for the molecular diagnosis of LCA.The study of patients affected with LCA could be divided on the basis of the clinical history of the disease, the existence of consanguinity, the primary mapping of the disease-locus, and the molecular screening of the gene of interest.

$100 \%$ (same gene). The identification of the diseasecausing gene is of great help to solve these ambiguities. From this point view, it is worth noting that in our series are included three families with both parents affected with LCA (Fig. 3). In the first two families, patients were studied before they married (Fig. 3A and B). In the first one, the woman carried a homozygous mutation in the GUCY2D gene (p.Phe565Ser) while her husband harbored a homozygous mutation in the RPGRIP1 gene (p.Gly746Glu). They were informed of absence of risk for descent and, indeed, they gave birth to two healthy children (Fig. 3A). In the second family, the woman carried compound heterozygote mutations in the GU. CY2D gene (p.Met1Ile, c.1957-1G>T). All genes were screened in her husband but no mutation was found. Again, an encouraging genetic counseling was given and, indeed, they gave birth to two healthy children (Fig. 3B). The third family was included in our series after the birth of two children. Both parents were unrelated but were born to consanguineous parents and were affected with LCA. No genetic counseling was done and the diagnosis of LCA was carried in the first child because his mother was already pregnant for the second time. Both children are affected with LCA. Both parents were found to carry different homozygous mutation in the CRB1 gene (p.Glu710Gln and p.Leu1107Arg respectively) and both children harbored compound heterozygote mutations (Fig. 3C).
Interestingly, $38 \%$ of the patients of our series originate from Mediterranean countries (68/179, Table 1). Among them, 27 (39.7\%) harbored a mutation in the GUCY2D gene. This might explain that in our panel, the proportion of patients harboring GUCY2D mutations is higher than in other series [Lotery et al., 2003]. This situation is comparable to that met for the rhodopsine gene in which the p.Pro23His mutation is prevalent in the United States $(12 \%$ of American autosomal dominant retinitis pigmentosa) [Dryja et al., 1990; Farrar et al., 1990] but not in Europe.

A wide allelic heterogeneity was found for all genes; however, some recurrent mutations were identified. For example, in the Mediterranean geographic subgroup, three distinct recurrent mutations were found in apparently unrelated families hailing from Maghreb, suggesting the existence of founder effects in North Africa (c.387delC, c.620delC, p.Ser448X).

Along the same lines, four unrelated families of Finnish origin were found to carry the same c.2943delG mutation not found in patients hailing from other countries worldwide [Hanein et al., 2002].

For more than half the patients of our series, the disease gene remains to be identified. In our hands, genome-wide search for homozygosity in large multiplex consanguineous families unlinked to any of the 10 LCA loci failed to identify a major locus. Subsequently, it is likely that many disease genes have to be identified, each 


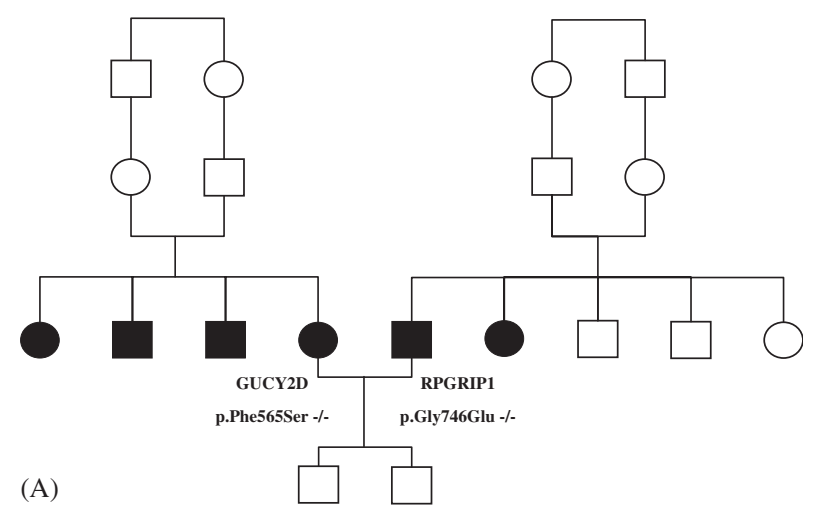

(B)
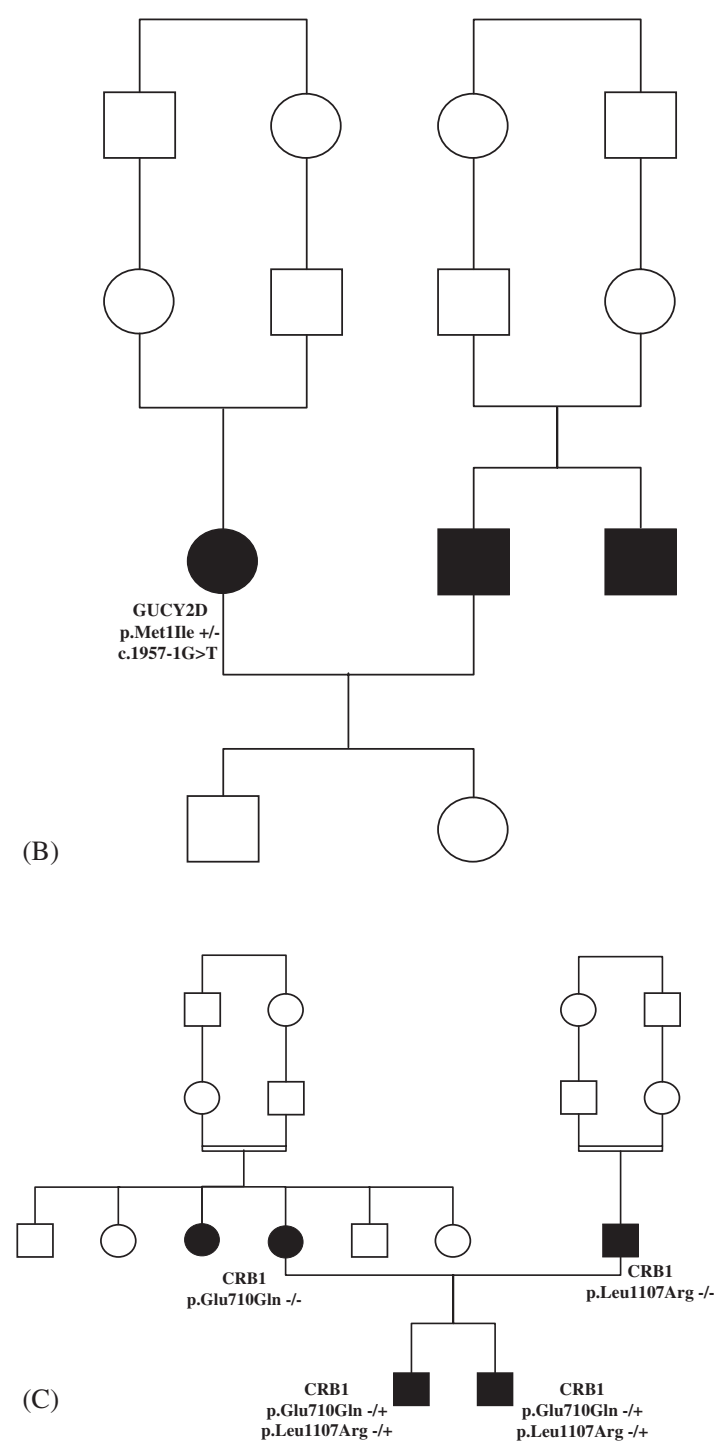

FIGURE 3. Pedigrees and segregation of disease-causing mutations in three LCA families (A, B, C, respectively) in which both parents are affected with LCA.

of them accounting for a small proportion of patients (unpublished results).

Whatever the gene (CRX apart), most patients (77/ $85,>90 \%)$ were found to be homozygote $(n=50)$ or compound heterozygotes $(\mathrm{n}=28)$ for mutations. Correct segregation of disease alleles was demonstrated in 71 cases in which family samples were available for study.

In seven unrelated patients, only one disease allele was identified (RPGRIP1, $\mathrm{n}=3$; RPE65, $\mathrm{n}=3$; GUCY2D, $\mathrm{n}=1$; respectively). Direct sequencing failed to identify a disease-associated mutation on the second allele. A possible digenism could have been considered to explain this. However, none of these seven patients were found to carry another mutation in one of the six other known LCA genes. In addition, none of the three patients with RPGRIP1 mutations were found to carry any alteration in the RPGRIP1 protein partner, RPGR, which mutations account for severe X-linked retinitis pigmentosa [Meindl et al., 1996]. Similarly, for the three unrelated patients with RPE65 mutations, we failed to identify any change in the lecithin retinol acyltransferase (LRAT), which also plays a crucial role in the retinoid cycle and in which alterations have been shown to account [Deigner et al., 1989], as RPE65 does, for some early-onset severe retinal dystrophies. Besides, it is admitted that in many recessive disorders, even with no evidence for non-allelic heterogeneity, only about $70 \%$ of the molecular defects can be found [Ducroq et al., 2002]. Therefore, it is likely that the undiscovered mutations lie in unscreened regions of the genes such as the promoter region (i.e., PAX6 [Okladnova et al., 1998a,b; Sander et al., 1999; Zheng et al., 2001]), intronic sequences, or $3^{\prime}$ untranslated regions (i.e., alpha-globin [Waggoner and Liebhaber, 2003]).

Along the same lines, it is worth noting that for small consanguineous families (one or two affected children) in which several chromosomal regions are found to be homozygotes, some can correspond to LCA loci. When the screening of the associated gene fails to identify the disease causing mutation, one cannot settle between homozygosity by random or a mutation lying in an unscreened region of the gene. Consequently, it is possible that the proportion of LCA patients related to one of the seven LCA genes will be higher than the $48 \%$ estimated in our series.

The growing number of LCA genes leads to growing difficulties in genotyping due to heavy time-consuming studies. However, genotyping remains essential before any therapeutic approach. It is why one of the only ways to get out of this difficult situation might be the establishment of phenotype-genotype correlations in order to direct molecular studies in a new patient.

The screening of LCA genes in our series evidenced a high prevalence of null alleles: (in decreasing order, CRX non included) $83.3 \%$ in RPGRIP1, 66.7\% in AIPL1, 62.7\% in GUCY2D, 57.9\% in RPE65, 36.1\% in CRB1, and $33.3 \%$ in TULP1. No correlation between the nature of the mutation and the phenotype in patients could be established within the same gene. From this point of view, it is worth noting that 21 different CRB1 mutations were identified in 18 patients of our series affected with a severe phenotype corresponding to the minimal criteria required for the diagnosis of LCA. Among these 21 mutations, three (p.Cys948Tyr, p.The745Met and p.Arg764Cys) were also identified in patients with 
autosomal recessive retinitis pigmentosa, characterized by a preservation of the para-arteriolar retinal pigment epithelium [den Hollander et al., 1999]. Nevertheless, the mutation of the second CRB1 allele was constantly different in the two, LCA and RP12, phenotypes.

However, in the past, a certain degree of clinical heterogeneity has been recognized in LCA [Traboulsi and Maumenee, 1995] but these subtle clinical differences have been subsequently neglected. In 1999, after the identification of the first two LCA genes, GUCY2D [Perrault et al., 1996] and RPE65 [Marlhens et al., 1997], we already demonstrated that mutations in these genes led to different functional outcome of the disease [Perrault et al., 1999]. More precisely, in a short series of 27 patients, 20 harboring mutations in GUCY2D and seven harboring mutations in RPE65, we showed that GUCY2D mutations were responsible for a congenital severe and stationary cone-rod dystrophy, while RPE65 mutations were responsible for a less severe and progressive rod-cone dystrophy [Perrault et al., 1999; Lorenz et al., 2000].

In the present study, our series of patients harboring mutations in one of the seven LCA genes has been enlarged to 85 of 179 patients $(47.5 \%)$. For these 85 patients, clinical data were obtained after rigorous new examinations allowing the division of the LCA patients into two groups according to the photoreceptor type first involved in the disease, cones or rods. Subsequently, a classification into four distinct clinical subtypes has been obtained on the basis of the progression course the disease, refraction error, the severity of the visual deficiency, and the aspect of the retina. Each clinical subtype is specific to one or two LCA genes. The two first subtypes belonging to the first group of patients are consistent with the traditional definition of LCA. Conversely, it is in the second group of patients, and especially in the second subtype, that the boundary between LCA and early onset severe retinal dystrophy (CSRD) becomes unclear, leading to the idea that in some cases, LCA can be considered as the extreme end of severity in the clinical spectrum of retinitis pigmentosa.

These findings allowed us to draw a clinical flowchart to direct the molecular analysis of selected LCA genes (Fig. 2). This flowchart is undoubtedly very helpful, as it lightens the heavy task of genotyping in a remarkably genetically heterogeneous condition.

In the past, linkage studies proved their power to map disease-causing genes in genetically homogeneous or slightly heterogeneous conditions. Conversely, in highly heterogeneous conditions such as LCA, linkage analyses undertaken to guide the molecular diagnosis in patients appear to be less useful in a significant proportion of cases despite informative markers because of the small size of the majority of familial cases (on average two affected sibs) and all the more so in consanguineous sporadic cases. Indeed, in small families, genome identity can be found by random, and in consanguineous cases, several homozygous chromosomal regions are found. Only very large families (four affected sibs or more) allow significant linkage to be obtained. In fact, these indirect studies are rather useful to exclude some loci and when several putative localizations are possible, only the phenotypegenotype correlations can help to select one locus more accurately. For this reason, we have drawn a decisional molecular diagnostic flowchart starting from the clinical history (Fig. 2). In the future, it would be ideal to have in all cases an informative clinical history (left part of the flowchart). In reality, situations are more complex because 1) in some cases, patients are too young (seen in the first months of life); 2) in some other cases, conversely, patients are seen too late in life and no clinical data are available for many reasons (dead parents, patients born in foreign countries with no clinical files); 3) accurate clinical data are difficult to obtain (parents refused repeated clinical examinations, existence of lack of understanding, language difficulties. etc.). In all three situations, the screening of all LCA genes (guided or not by linkage studies) remains topical.

\section{REFERENCES}

Deigner PS, Law WC, Canada FJ, Rando RR. 1989. Membranes as the energy source in the endergonic transformation of vitamin A to 11-cis-retinol. Science 26;244:968-971.

den Hollander AI, ten Brink JB, de Kok Y JM, van Soest S, van den Born LI, van Driel MA, van de Pol D Jr, Payne AM, Bhattacharya SS, Kellner U, Hoyng CB, Westerveld A, Brunner HG, Bleeker-Wagemakers EM, Deutman AF, Heckenlively JR, Cremers FPM, Bergen A. 1999. Mutations in a human homologue of Drosophila crumbs cause retinitis pigmentosa (RP12). Nature Genet 23:217-221.

den Hollander AI, Heckenlively JR, van den Born LI, de Kok YJ, van der Velde-Visser SD, Kellner U, Jurklies B, van Schooneveld MJ, Blankenagel A, Rohrschneider K, Wissinger B, Cruysberg JR, Deutman AF, Brunner HG, Apfelstedt-Sylla E, Hoyng CB, Cremers FP. 2001. Leber congenital amaurosis and retinitis pigmentosa with Coats-like exudative vasculopathy are associated with mutations in the crumbs homologue 1 (CRB1) gene. Am J Hum Genet 69:198-203.

Dharmaraj S, Li Y, Robitaille JM, Silva E, Zhu D, Mitchell TN, Maltby LP, Baffoe-Bonnie AB, Maumenee IH. 2000. A novel locus for Leber congenital amaurosis maps to chromosome 6q. Am J Hum Genet 66:319-326.

Dib C, Faure S, Fizames C. 1996. A comprehensive genetic map of the human genome based on 5,264 microsatellites. Nature 380:152-154.

Dryja TP, McGee TL, Hahn LB, Cowley GS, Olsson JE, Reichel E, Sandberg MA, Berson EL. 1990. Mutations within the rhodopsin gene in patients with autosomal dominant retinitis pigmentosa. N Engl J Med 323:1302-1307.

Dryja TP, Adams SM, Grimsby JL, McGee TL, Hong DH, Li T, Andreasson S, Berson EL. 2001. Null rpgrip1 alleles in patients with leber congenital amaurosis. Am J Hum Genet 68: 1295-1298.

Ducroq D, Rozet JM, Gerber S, Perrault I, Barbet D, Hanein S, Hakiki S, Dufier JL, Munnich A, Hamel C, Kaplan J. 2002. The ABCA4 gene in autosomal recessive cone-rod dystrophies. Am J Hum Genet 71:1480-1482.

El-Shanti H, Al-Salem M, El-Najjar M, Ajlouni K, Beck J, Sheffiled VC, Stone EM. 1999. A nonsense mutation in the retinal specific guanylate cyclase gene is the cause of Leber congenital amaurosis in a large inbred kindred from Jordan. J Med Genet 36:862-865. 
Farrar GJ, Kenna P, Redmond R, McWilliam P, Bradley DG, Humphries MM, Sharp EM, Inglehearn CF, Bashir R, Jay M. 1990. Autosomal dominant retinitis pigmentosa: absence of the rhodopsin proline-histidine substitution (codon 23) in pedigrees from Europe. Am J Hum Genet 47:941-945.

Franceschetti A, Dieterle P. 1954. Importance diagnostique et prognostique de l'électrorétinogramme (ERG) dans les dégénérescences tapéto-rétiniennes avec rétrécissement du champ visuel et héméralopie. Confin Neurol 14:184-186.

Freund CL, Wang QL, Chen S, Muskat BL, Wiles CD, Sheffield VC, Jacobson SG, McInnes RR, Zack DJ, Stone EM. 1998. De novo mutations in the CRX homeobox gene associated with Leber congenital amaurosis. Nat Genet 18:311-312.

Gerber S, Perrault I, Hanein S, Barbet F, Ducroq D, Ghazi I, Martin-Coignard D, Leowski C, Homfray T, Dufier JL, Munnich A, Kaplan J, Rozet JM. 2001. Complete exon-intron structure of the RPGR-interacting protein (RPGRIP1) gene allows the identification of mutations underlying Leber congenital amaurosis. Eur J Hum Genet 9:561-571.

Gerber S, Perrault I, Hanein S, Shalev S, Zlotogora J, Barbet F, Ducroq D, Dufier J, Munnich A, Rozet J, Kaplan J. 2002. A novel mutation disrupting the cytoplasmic domain of CRB1 in a large consanguineous family of Palestinian origin affected with Leber congenital amaurosis. Ophthalmic Genet 23:225-235.

Gu SM, Thompson DA, Srikumari CR, Lorenz B, Finckh U, Nicoletti A, Murthy KR, Rathmann M, Kumaramanickavel G, Denton MJ, Gal A. 1997. Mutations in RPE65 cause autosomal recessive childhood-onset severe retinal dystrophy. Nat Genet 17:194-197.

Hanein S, Perrault I, Olsen P, Lopponen T, Hietala M, Gerber S, Jeanpierre M, Barbet F, Ducroq D, Hakiki S, Munnich A, Rozet JM, Kaplan J. 2002. Evidence of a founder effect for the RETGC1 (GUCY2D) 2943DelG mutation in Leber congenital amaurosis pedigrees of Finnish origin. Hum Mutat 20:322-323.

Jacobson SG, Cideciyan AV, Huang Y, Hanna DB, Freund CL, Affatigato LM, Carr RE, Zack DJ, Stone EM, McInnes RR. 1998. Retinal degenerations with truncation mutations in the cone-rod homeobox (CRX) gene. Invest Ophthalmol Vis Sci 39:2417-2426.

Kaplan J, Bonneau D, Frezal J, Munnich A, Dufier JL. 1990. Clinical and genetic heterogeneity in retinitis pigmentosa. Hum Genet 86:635-642.

Keen TJ, Mohamed MD, McKibbin M, Rashid Y, Jafri H, Maumenee IH, Inglehearn CF. 2003. Identification of a locus (LCA9) for Leber's congenital amaurosis on chromosome $1 \mathrm{p} 36$. Eur J Hum Genet 11:420-423.

Leber T. 1869. Retinitis pigmentosa und angeborene Amaurose. Albrecht von Graefes Arch Ophthal 15:1-25.

Lorenz B, Gyurus P, Preising M, Bremser D, Gu S, Andrassi M, Gerth C, Gal A. 2000. Early-onset severe rod-cone dystrophy in young children with RPE65 mutations. Invest Ophthalmol Vis Sci 41:2735-2742.

Lotery AJ, Jacobson SG, Fishman GA, Weleber RG, Fulton AB, Namperumalsamy P, Heon E, Levin AV, Grover S, Rosenow JR, Kopp KK, Sheffield VC, Stone EM. 2001. Mutations in the CRB1 gene cause Leber congenital amaurosis. Arch Ophthalmol 119:415-420.

Lotery AJ, Jacobson SG, Weleber RG, Iannaccone A, Namperumalsamy P, Fishman GA, Levin A, Lam BL, Heon E, Stone EM. 2003. Prevalence of mutations in the RPE65, CRX, AIPL1, TULP1, GUCY2D and CRB1 genes in Leber congenital amaurosis. Invest Ophthalmol Vis Sci 44:E-2301.
Marlhens F, Bareil C, Griffoin JM, Zrenner E, Amalric P, Eliaou C, Liu SY, Harris E, Redmond TM, Arnaud B, Claustres M, Hamel CP. 1997. Mutations in RPE65 cause Leber's congenital amaurosis. Nat Genet 17:139-141.

Meindl A, Dry K, Herrmann K, Manson F, Ciccodicola A, Edgar A, Carvalho MR, Achatz $\mathrm{H}$, Hellebrand $\mathrm{H}$, Lennon $\mathrm{A}$, Migliaccio C, Porter K, Zrenner E, Bird A, Jay M, Lorenz B, Wittwer B, D'Urso M, Meitinger T, Wright A. 1996. A gene (RPGR) with homology to the RCC1 guanine nucleotide exchange factor is mutated in X-linked retinitis pigmentosa (RP3). Nat Genet 13:35-42.

Morimura H, Fishman GA, Grover SA, Fulton AB, Berson EL, Dryja TP. 1998. Mutations in the RPE65 gene in patients with autosomal recessive retinitis pigmentosa or Leber congenital amaurosis. Proc Natl Acad Sci USA 95:3088-3093.

North MA, Naggert JK, Yan Y, Noben-Trauth K, Nishina PM. 1997. Molecular characterization of TUB, TULP1, and TULP2, members of the novel tubby gene family and their possible relation to ocular diseases. Proc Natl Acad Sci USA 94:3128-3133.

Okladnova O, Syagailo YV, Mossner R, Riederer P, Lesch KP. 1998a. Regulation of PAX-6 gene transcription: alternate promoter usage in human brain. Brain Res Mol Brain Res 60:177-192.

Okladnova O, Syagailo YV, Tranitz M, Stober G, Riederer P, Mossner R, Lesch KP. 1998b. A promoter-associated polymorphic repeat modulates PAX-6 expression in human brain. Biochem Biophys Res Commun 248:402-405.

Perrault I, Rozet JM, Calvas P, Gerber S, Camuzat A, Dollfus H, Chatelin S, Souied E, Ghazi I, Leowski C, Bonnemaison M, Le Paslier D, Frezal J, Dufier JL, Pittler S, Munnich A, Kaplan J. 1996. Retinal-specific guanylate cyclase gene mutations in Leber's congenital amaurosis. Nat Genet 14:461-464.

Perrault I, Rozet JM, Ghazi I, Leowski C, Bonnemaison M, Gerber S, Ducroq D, Cabot A, Souied E, Dufier JL, Munnich A, Kaplan J. 1999. Different functional outcome of retGC1 and RPE65 gene mutations in Leber congenital amaurosis. Am J Hum Genet 64:1225-1228.

Perrault I, Rozet JM, Gerber S, Ghazi I, Ducroq D, Souied E, Leowski C, Bonnemaison M, Dufier JL, Munnich A, Kaplan J. 2000. Spectrum of retGC1 mutations in Leber's congenital amaurosis. Eur J Hum Genet 8:578-582.

Perrault I, Hanein S, Gerber S, Barbet F, Dufier J-L, Munnich A, Rozet J-M, Kaplan J. 2003. Evidence of autosomal dominant Leber congenital amaurosis (LCA) underlain by a CRX heterozygote null allele. J Med Genet 40:E90.

Rozet JM, Perrault I, Gerber S, Hanein S, Barbet F, Ducroq D, Souied E, Munnich A, Kaplan J. 2001. Complete abolition of the retinal-specific guanylyl cyclase (retGC-1) catalytic ability consistently leads to leber congenital amaurosis (LCA). Invest Ophthalmol Vis Sci 42:1190-1192.

Sander T, Syagailo Y, Samochowiec J, Okladnova O, Lesch KP, Janz D. 1999. Association analysis of a regulatory promoter polymorphism of the PAX-6 gene with idiopathic generalized epilepsy. Epilepsy Res 36:61-67.

Simovich MJ, Miller B, Ezzeldin H, Kirkland BT, McLeod G, Fulmer C, Nathans J, Jacobson SG, Pittler SJ. 2001. Four novel mutations in the RPE65 gene in patients with Leber congenital amaurosis. Hum Mutat 18:164.

Sohocki MM, Bowne SJ, Sullivan LS, Blackshaw S, Cepko CL, Payne AM, Bhattacharya SS, Khaliq S, Qasim Mehdi S, Birch DG, Harrison WR, Elder FF, Heckenlively JR, Daiger SP. 2000a. Mutations in a novel photoreceptor-pineal gene on $17 \mathrm{p}$ cause Leber congenital amaurosis (LCA4). Nat Genet 24:79-83. 
Sohocki MM, Perrault I, Leroy BP, Payne AM, Dharmaraj S, Bhattacharya SS, Kaplan J, Maumenee IH, Koenekoop R, Meire FM, Birch DG, Heckenlively JR, Daiger SP. 2000b. Prevalence of AIPL1 mutations in inherited retinal degenerative disease. Mol Genet Metab 70:142-150.

Stockton DW, Lewis RA, Abboud EB, Al-Rajhi A, Jabak M, Anderson KL, Lupski JR. 1998. A novel locus for Leber congenital amaurosis on chromosome 14q24. Hum Genet 103:328-333.

Swaroop A, Wang QL, Wu W, Cook J, Coats C, Xu S, Chen S, Zack DJ, Sieving PA. 1999. Leber congenital amaurosis caused by a homozygous mutation (R90W) in the homeodomain of the retinal transcription factor CRX: direct evidence for the involvement of CRX in the development of photoreceptor function. Hum Mol Genet 8:299-305.
Traboulsi EI, Maumenee IH. 1995. Photoaversion in Leber's congenital amaurosis. Ophthalmic Genet 16:27-30.

Tzekov RT, Liu Y, Sohocki MM, Zack DJ, Daiger SP, Heckenlively JR, Birch DG. 2001. Autosomal dominant retinal degeneration and bone loss in patients with a 12-bp deletion in the CRX gene Invest. Ophthalmol Vis Sci 42:1319-1327.

Wardenburg PJ, Schappert-Kimmijser J. 1963. On various recessive biotypes of Leber cobgenital amaurosis. Acta Ophthalmol 41:317-320.

Waggoner SA, Liebhaber SA. 2003. Regulation of alpha-globin mRNA stability. Exp Biol Med 228:387-395.

Zheng JB, Zhou YH, Maity T, Liao WS, Saunders GF. 2001. Activation of the human PAX6 gene through the exon 1 enhancer by transcription factors SEF and Sp1. Nucleic Acids Res 29:4070-4078. 